HORTSCIENCE 26(5):592-594. 1991

\title{
Chloroplast Density in Guard Cells of Leaves of Anther-derived Potato Plants Grown in Vitro and in Vivo
}

\author{
Chongkhohao Singsit ${ }^{1}$ and Richard E. Veilleux \\ Department of Horticulture, Virginia Polytechnic Institute and State \\ University, Blacksburg, VA 24061
}

Additional index words. monoploid, Solarium, androgenesis, haploid

\begin{abstract}
A technique was developed to count chloroplasts in guard cell pairs of leaflets of anther-derived potato (Solanum phureja Juz. and Buk) plants grown in vitro to determine how they compare to chloroplast counts for similar plants grown in vivo. The mean chloroplast density in guard cells of in vitro leaflets was visualized using fluorescein diacetate (FDA) stain and observed under a microscope. A consistently higher mean was observed for in vitro than for in vivo plants but monoploid could readily be distinguished from diploid regenerants whether grown in vitro or in vivo. A rapid method of ploidy estimation based on chloroplast counts on in vitro leaflets may hasten identification of monoploids to be used for in vitro applications to genetic reconstruction of potato.
\end{abstract}

Development of a convenient, fast, and accurate procedure to determine the ploidy status of anther-derived plants would facilitate early identification of monoploids and hasten disposal of undesirable higher ploidy levels that generally have comprised the majority of anther-derived plants (Veilleux et al., 1985; Wenzel and Uhrig, 1981). Chromosome counts from mitotic cells in root tips or meiotic cells in flower buds are accurate but generally require that plantlets be accli-

Received for publication 18 Oct. 1990. This work was supported in part by state and Hatch Act funds (project 132530). We wish to thank Suzanne Piovano and Joyce Shelton for their technical and secretarial assistance. The cost of publishing this paper was defrayed in part by the payment of page charges. Under postal regulations, this paper therefore must be hereby marked advertisement solely to indicate this fact.

'Present address: Dept. of Horticulture, Coastal Plain Experiment Station, Univ. of Georgia, Tifton, GA 31793. mated to in vivo conditions, a process during which spontaneous chromosome doubling has been documented (Karp et al., 1984; Wenzel and Uhrig, 1981). In addition, anther-derived monoploid potato plants are usually weak and difficult to establish in vivo.

Frandsen (1968) estimated the number of chromosomes in potato using the mean number of chloroplasts per pair of guard cells. A correlation between the mean number of chloroplasts and the number of chromosomes was observed; the mean was in-

Table 1. Analysis of variance for mean chloroplast density of 11 anther-derived potato plants from two environments.

\begin{tabular}{lrc}
\hline \hline Source & df & Mean square \\
\hline Replication & 2 & \\
Environment (E) & 1 & $25.72^{* *}$ \\
Genotype (G) & 10 & $51.30^{* *}$ \\
G $\times$ E interaction & 10 & $1.91^{* *}$ \\
Error & 42 & 0.39 \\
\hline
\end{tabular}

**Significant at $P=0.01$. 
Table 2. Pooled means \pm SE for chloroplast density of 11 anther-derived potato plants from two environments.

\begin{tabular}{lcrr}
\hline \hline & $\begin{array}{c}\text { Combined } \\
\text { menotype }\end{array}$ & \multicolumn{2}{c}{ Mean $( \pm$ SE) chloroplast density } \\
\cline { 4 - 4 } AX-87-13 & $16.9 \mathrm{a}$ & $17.1 \pm 0.4$ & In vivo \\
AX-87-31 & $16.3 \mathrm{ab}$ & $18.3 \pm 0.5$ & $16.7 \pm 0.6$ \\
$\mathrm{AX}-87-20$ & $16.3 \mathrm{ab}$ & $17.1 \pm 0.5$ & $14.3 \pm 0.1$ \\
$\mathrm{AX}-87-26$ & $15.9 \mathrm{~b}$ & $17.0 \pm 0.3$ & $15.4 \pm 0.1$ \\
$\mathrm{AX}-87-30$ & $15.4 \mathrm{bc}$ & $16.3 \pm 0.6$ & $14.7 \pm 0.2$ \\
$\mathrm{AX}-87-10$ & $14.9 \mathrm{bcd}$ & $15.2 \pm 0.5$ & $14.5 \pm 0.2$ \\
$\mathrm{AX}-87-12$ & $14.2 \mathrm{~d}$ & $14.2 \pm 0.7$ & $14.6 \pm 0.1$ \\
$\mathrm{AX}-87-32$ & $10.4 \mathrm{e}$ & $10.6 \pm 0.2$ & $14.1 \pm 0.1$ \\
$\mathrm{AX}-87-1$ & $10.3 \mathrm{e}$ & $10.8 \pm 0.2$ & $10.2 \pm 0.2$ \\
$\mathrm{AX}-87-9$ & $9.9 \mathrm{e}$ & $10.6 \pm 0.6$ & $9.9 \pm 0.2$ \\
AX-87-37 & $9.7 \mathrm{e}$ & $9.8 \pm 0.3$ & $9.3 \pm 0.2$ \\
\hline
\end{tabular}

${ }^{2}$ Mean separation within columns by Student-Newman-Keul's test, $P=0.05$.

creased by a factor of 1.8 to 1.9 after each doubling of the chromosome number. Since then, the mean number of chloroplasts per pair of guard cells has routinely been used to estimate the number of chromosomes of anther-derived potato plants in vivo (Cappadocia et al., 1984; Singsit and Veilleux, 1988; Uhrig and Salamini, 1987; Veilleux et al., 1985; Wenzel and Uhrig, 1981). Although the procedure has also been used on in vitro plantlets (Jacobs and Yoder, 1989), a comparison between in vivo and in vitro chloroplast counts has not been published. In vitro leaflets are morphologically distinct from in vivo leaflets (Pehu et al., 1987), and the photosynthetic apparatus might be less completely developed. The objectives of this study were to: 1) develop a reliable technique for counting chloroplasts in guard cell pairs from anther-derived in vitro potato plantlets, and 2) compare the results of in vitro chloroplast counts with those of in vivo counts to estimate ploidy of the same regenerants.

A replicated study was conducted for 11 plants derived by anther culture of a single clone of potato. The chloroplast density per guard cell pair of anther-derived plants was studied in two environments, in vitro and in vivo. Plant materials were propagated by nodal cuttings and grown on a basal medium (Murashige and Skoog, 1962) for 4 to 6 months. Rooted cuttings were established in vivo following the procedures of Singsit and Veilleux (1988). Plants were grown in a completely randomized design with three replications in each environment. The mean number of chloroplasts was obtained from ten guard cell pairs per replicate per regenerant.

Chloroplasts were counted from two or three leaflets removed from in vitro plantlets. Each leaflet was placed on a glass slide, adaxial side up, and the mesophyll tissues were removed by slight scraping with a spatula and camel hair brush to expose the lower epidermis, which was stained with fluorescein diacetate (FDA). A $0.5 \%$ FDA solution was prepared in acetone, stored at $0 \mathrm{C}$, and adjusted to $\approx 0.01 \%$ (by volume) by diluting one drop FDA stain with 20 to 25 drops of distilled water. A cover slip was mounted on the stained epidermis, which was observed at $\times 400$ magnification under a Zeiss $(\mathrm{Ob}-$ erkochen, Germany) fluorescent microscope equipped with a fluorescein isothiocyanate (FITC) filter combination. The chloroplasts that absorbed the FDA appeared green, whereas unstained chloroplasts were red due to autofluorescence of chlorophyll. For greenhouse-grown plants, an epidermal strip was peeled from a leaf with forceps, dipped in distilled water, covered with a cover slip, and observed under a fluorescent microscope according to Frandsen (1968) using the same FITC filter combination but without stain. Data were analyzed using the General Linear Models procedures of the Statistical Analysis System (SAS, 1985), and the resulting means were compared for both environments.

The analysis of variance (Table 1) revealed significant differences between environments, among genotypes, and for genotype $\times$ environment (GE) interaction for chloroplast density. The 11 genotypes were classified into two distinct groups, one consisting of seven clones with a mean chloroplast density per guard cell pair of 14.8 and one consisting of four clones with a mean chloroplast density of 9.5. Classification of the larger group as diploid and the smaller as monoploid was based on previous accounts (Frandsen, 1968; Karp et al., 1984; Pehu et al., 1987) where a chloroplast density per guard cell pair below a mean of 12 has consistently been found to correspond to monoploid plants. The ratio between the mean chloroplast density of the presumed monoploid and diploid groups was 1.6; this was slightly lower than that reported by Frandsen (1.8 to 1.9) but consistent with other reports (Karp et al., 1984; Pehu et al., 1987). Potential monoploids were distinctly different from diploids and were characterized by fewer chloroplasts in both environments. Slightly more chloroplasts per guard cell pair were observed for in vitro than for in vivo plants (Table 2); the discrepancy is attributed largely to environmental differences under which the plants were grown. In the in vitro environment, leaves are much smaller than in vivo; perhaps a higher chloroplast density per guard cell pair is a compensatory mechanism to adjust for smaller leaf area. The occurrence of significant GE interaction can be interpreted to mean that some regenerants were more stable (e.g., AX-87-12) across environments than others (e.g., AX-87-31). Even though significant differences were observed within the diploid group, the mean chloroplast density remained within the previously estimated diploid range (Karp et al., 1984; Pehu et al., 1987); the differences maybe attributed to the mode of origin of the diploids, i.e., from $2 \mathrm{n}$ pollen or. somatic tissues (heterozygous) vs. spontaneous chromosome doubling (homozygous). A highly positive correlation $(r=0.94, P<0.01)$ for mean chloroplast density between in vitro and in vivo means allows in vitro chloroplast counts to be used as a reliable ploidy estimate for anther-derived plants.

Guard cells are representative of the $\mathrm{L}$, tissue layer, whereas microsporocytes and meristematic ceils in root tips represent the $\mathrm{L}_{2}$ and $\mathrm{L}_{3}$ layers, respectively (Williams, 1968). The epidermis is formed from $L_{1}$, the cortex and eventually the pollen and embryosac mother cells are formed from $\mathrm{L}_{2}$ and the stele is formed from $\mathrm{L}_{3}$. In chimeric studies, it is usually only $\mathrm{L}_{1}$ and $\mathrm{L}_{2}$ layers that are important. Hence, a discrepancy among ploidy estimates might reflect the chimeric nature of an anther-derived plant rather than spontaneous chromosome doubling. Examination of only a single tissue will not identify chimerism, and some of these plants could be chimeric. For two of the four monoploids (AX-87-32, AX-87-37), L ploidy was determined by root tip chromosome counts of in vivo plants and found to correspond to the monoploid chromosome number $(2 \mathrm{n}=\mathrm{x}=$ 12).

FDA stain was necessary only for in vitro leaflets. Poor cuticle and chlorophyll development prevented accurate counts by autofluorescence of chlorophyll due to interference from chloroplasts in mesophyll cells, which had not been completely removed. The FDA stain permitted easy visualization and counting of guard cell chloroplasts, but was unnecessary for in vivo leaflets from which intact epidermal peels with brightly autofluorescing chloroplasts could be easily removed. We later found that in vitro leaflets could be handled more quickly and easily by simply painting the diluted FDA stain onto the abaxial epidermis with a fine camel hair brush, placing the intact leaflet in a drop of water, and covering it with a cover slip without any attempt to separate the mesophyll from the epidermis, Five minutes after staining, the guard cell chloroplasts would fluoresce bright green against a red background of autofluorescing chloroplasts in mesophyll cells. Counts could then be made quickly before the FDA penetrated through the mesophyll tissue. Bright fluorescence of chloroplasts was obtained for both in vivo and in vitro plants. The different staining procedures could not modify the chloroplast density; they simply allowed accurate counts to be obtained for each situation. We found that chloroplasts were much easier to score accurately using these methods under a fluorescent light source than with $\mathrm{AgNO}_{3}$ stain under brightfield (Frandsen, 1968; Jacobs and Yoder, 1989).

In the past, various plant attributes, such as stomatal guard cell length (Barrino and Powell, 1988), morphological characters (Pehu et al., 1987; Santos and Handro, 1983), and cytometric analysis of DNA content 
(Owen et al., 1988; Sharma et al., 1983; Uijtewaal, 1987), have been used to estimate the ploidy status of plants regenerated from tissue cultures. However, most of these techniques are either labor-intensive or require expensive equipment. However, in vitro chloroplast counting allows rapid ploidy screening of many plants without special equipment or skills and is well correlated with ploidy estimates from in vivo chloroplast counts. Nevertheless, confirmation of the actual ploidy level in indistinct cases can only be ascertained by chromosome counting. An in' vitro screen may be useful for early identification of potential monoploids, especially when establishment in vivo is unnecessary, e.g., in protoplast manipulation and genetic transformation.

\section{Literature Cited}

Barrino, E.M. and W. Powell. 1988. Stomata] guard cell length as an indicator of ploidy in microspore-derived plants of barley. Genome 30:158-160.

Cappadocia, M., D.S.K. Cheng, and R. LudlumSimonette. 1984. Plant regeneration from in vitro culture of anthers of Solanum chacoense Bitt. and interspecific diploid hybrids of S. tuberosum L. $\times$ S. chacoense Bitt. Theor. Appl. Genet. 69:139-143.

Frandsen, N.O. 1968. Die Plastidenzahl als Merkmal bei der Kartoffel. Theor. Appl. Genet. 38:153-167.

Jacobs, J.P. and J.I. Yoder. 1989. Ploidy levels in transgenic tomato plants determined by chloroplast number. Plant Cell Rpt. 7:662-664.

Karp, A., R. Risiott, M.G.K. Jones, and S.W.J. Bright. 1984. Chromosome doubling in monohaploid and dihaploid potatoes by regeneration from cultured leaf explants. Plant Cell Tissue Organ Cult. 3:363-373.

Murashige, T. and F. Skoog. 1962. A revised medium for rapid growth and bioassays with tobacco tissue culture. Physiol. Plant. 15:473497.

Owen, H. R., R.E. Veilleux, D. Levy, and D.L. Ochs. 1988. Environmental, genotypic, and ploidy effects on endopolyploidization within a genotype of Solarium phureja and its derivatives. Genome 30:506-510.

Pehu, E., R.E. Veilleux, and K.W. Hilu. 1987. Cluster analysis of anther-derived plants of $\mathrm{So}$ larium phureja (Solanaceae) based on morphological characters. Amer. J. Bet. 74:47-52.

Santos, R.F. and W. Handro. 1983. Morphological patterns in Petunia hybrida plants regenerated from tissue cultures differing by their ploidy. Theor. Appl. Genet. 66:55-60.

SAS Institute. 1985. SAS user's guide: Statistics version 5 ed. SAS Institute, Inc., Cary, N.C.

Sharma, D. P., E. Firoozabady, N.M. Ayers, and D. Galbraith. 1983. Improvement of anther culture in Nicotiana: Media, cultural conditions and flow cytometric determination of ploidy levels. Z. Pflanzenphysiol. 111:441-451.

Singsit, C. and R.E. Veilleux. 1988. Intra- and interspecific transmission of androgenetic competence in diploid potato species. Euphytica 43:105-112.

Uhrig, H. and F. Salamini. 1987. Dihaploid plant production from $4 \mathrm{x}$ genotypes of potato by the use of efficient anther plants producing tetraploid strains (4x EAPP clones) -Proposal of a breeding methodology. Plant Breeding 98:228235.

Uijtewaal, B.A. 1987. Ploidy variability in green- house cultured and in vitro propagated potato Solarium phureja monohaploids $(2 \mathrm{n}=1 \mathrm{x}=$ 12 ) as determined by flow cytometry. Plant Cell Rpt. 6:252-255.

Veilleux, R. E., J. Booze-Daniels, and E. Pehu. 1985. Anther culture of a $2 \mathrm{n}$ pollen producing clone of Solanum phureja Juz. \& Buk. Can. J.
Genet. Cytol. 27:559-564.

Wenzel, G. and H. Uhrig. 1981. Breeding for nematode and virus resistance in potato via anther culture. Theor. Appl. Genet. 59:333-340.

Williams, W. 1968. Genetical principles and plant breeding. BlackWell Scientific Publications, Oxford. p. 176-183. 